\section{Comparison of tuberculosis indicators after implementation of the clinical protocol for tuberculosis and HIV management in Iranian prisons: a quasi-experimental study}

Tuberculosis indicators in Iranian prisons

\author{
SeyedAhmad SeyedAlinaghi
}

Iranian Research Center for HIV/AIDS, Iranian Institute for Reduction of High-Risk Behaviors, Tehran University of Medical Sciences, Tehran, Iran

Behnam Farhoudi

Social Determinants of Health Research Center, Amir-Almomenin Hospital, Tehran Medical Sciences Branch, Islamic Azad University, Tehran, Iran

Bahar Ataeinia

Iranian Research Center for HIVIAIDS, Iranian Institute for Reduction of High-Risk Behaviors, Tehran University of Medical Sciences, Tehran, Iran

Omid Dadras

Department of Global Health and Socioepidemiology, Kyoto University, Kyoto, Japan Mostafa Hosseini

Department of Epidemiology and Biostatistics,

School of Public Health, Tehran University of Medical Sciences, Tehran, Iran

Sirous Jafari and Elham Mazaheri-Tehrani

Iranian Research Center for HIVIAIDS, Iranian Institute for Reduction of High-Risk Behaviors, Tehran University of Medical Sciences, Tehran, Iran

Ramin Alasvand and Mohammad Shahbazi

Health and Treatment Office of Iranian Prisons Organization, Tehran, Iran, and

Minoo Mohraz

Iranian Research Center for HIV/AIDS, Iranian Institute for Reduction of High-Risk Behaviors, Tehran University of Medical Sciences, Tehran, Iran

(C) SeyedAhmad SeyedAlinaghi, Behnam Farhoudi, Bahar Ataeinia, Omid Dadras, Mostafa Hosseini, Sirous Jafari, Elham Mazaheri-Tehrani, Ramin Alasvand, Mohammad Shahbazi and Minoo Mohraz. Published in the Journal of Health Research. Published by Emerald Publishing Limited. This article is published under the Creative Commons Attribution (CC BY 4.0) license. Anyone may reproduce, distribute, translate and create derivative works of this article (for both commercial and non-commercial purposes), subject to full attribution to the original publication and authors. The full terms of this license may be seen at $\mathrm{http} / /$ creativecommons.org/licences/by/4.0/legalcode

The authors thank all staff in the prisons for their help.

Conflict of interests: The authors declare that they have no competing interests.

Funding: This project was founded by UNODC in Iran.

This project was founded by WHO office in Iran (a resource of fund from Global Fund to fight AIDS TB, and Malaria in prison organization). We also thank all staff of the Great Tehran Prison for their help. 
JHR

34,6

\begin{abstract}
Purpose - The aim of this study was to compare the defined indicators of tuberculosis (TB) control program in the intervention and control prisons, after implementation of the national clinical protocol for TB and HIV management in Iranian prisons, suggesting active health service provision in all steps of service provision.

Design/methodology/approach - This was quasi-experimental study conducted among inmates of two prisons in Iran. Great Tehran prison was purposively chosen as the intervention prison and Karaj prison was purposively chosen as control prison as well. Intervention and control prisons were compared in terms of the TB indicators within three periods (before intervention, during implementation and follow-up period) from October 2013 to June 2014.
\end{abstract}

Findings - Number of inmates with TB symptoms who underwent TB workup was four times more in intervention prison compared to control prison (9.3 vs 2.5 cases out of 1,000 inmates per month in the case prison compared to the control prison). Such difference was also significant in the intervention prison, comparing before and during the intervention period. The patient finding in case prison increased significantly after the intervention (223.6 vs 81.8 cases out of 100,000 inmates per year). The number of TB cases who received HIV testing increased from 50 to $100 \%$.

Originality/value - Active health service provision has significantly improved indicators in the intervention prison. The authors recommend implementation of this guideline in all prisons of Iran. Integration of other diseases with high burden among prisoners is also recommended in the active health services provision.

Keywords Prison, Tuberculosis, HIV management, Iran

Paper type Research paper

\title{
Introduction
}

In 2017, the number of incarcerated people in Iran was about 230,000. Furthermore, the prisoner turnover rate is around 2.5-3 times more than the prison population each year [1]. Tuberculosis (TB) infection is usually more prevalent among incarcerated people worldwide, irrespective of the economic status and the TB burden in the country. According to the World Health Organization (WHO), the prevalence of TB in prisons is about 10- to 100-fold higher than that of the general population [2]. In prisons located in developing countries, TB is the most common cause of death and a major health problem [3].

There are different risk factors for TB infection in prisons. They include overcrowding, poor ventilation, malnutrition, late case detection, inadequate treatment of infectious cases, high turnover rate of prisoners, poor implementation of TB infection control measures and close contact with individuals who are already at a higher risk of TB infection (former prisoners, drug users, homeless people and illegal immigrants from endemic areas) [3]. These risk factors increase the risk of transmission of airborne infections such as TB. Moreover, the high prevalence of risky behaviors such as unprotected sex, rape and unsafe injecting practices contribute to the higher risk of HIV, which dramatically increases the risk of reactivation of latent TB infection (LTBI) [4].

$\mathrm{HIV}$ is the most potent risk factor for TB infection. Annually, an estimated $8 \%$ of TB cases occur among HIV patients worldwide [5]. Prisons are considered as a reservoir facilitating Mycobacterium tuberculosis (MTB) transmission among the general population through prison staff, visitors and released inmates $[2,3,6]$. The estimated rate of TB infection in the general population due to the transmission in prisons is $8.5 \%$ in high-income countries and $6.3 \%$ in middle- or low-income countries $[2,3]$

TB infection control depends on minimizing the risk of TB transmission. This is mainly achieved by early detection and effective treatment of TB cases [5]. Effective TB control programs protect both inmates and the general population [7]. In addition, prisons are valuable places to provide health services to prisoners. However, the evidence indicated that this chance is frequently missed [7].

Active health service provision (AHSP), a new model for Iranian health service provision, has previously been described in detail in national clinical guidelines [8] and aims to restructure the health service delivery system to actively and effectively find, treat and follow TB patients. The 
aim of this study was to compare the defined indicators of TB in the intervention and control prisons after implementation of AHSP for TB and HIV management in Iranian prisons.

\section{Materials and methods \\ Study subjects and participants}

The detailed method of this study was described elsewhere [9]. Briefly, AHSP includes all aspects of TB diagnosis, treatment and care services. Guidelines consist of different topics concerning TB such as TB active or passive case finding in prison, confirming the diagnosis of active TB, treatment of active TB in prison, monitoring of TB treatment, drug interactions and adverse effects, diagnosis and treatment of latent TB in prison, patient's education, control of TB transmission in prison and patient follow-up after the release of the offender. Likewise, HIV topics included HIV testing at prison administration and in units, consultation after HIV testing and subsequent confirmation, evaluation of when to start antiretroviral therapy (ART), synchronization of ART and TB medications in prison, preventive therapy with co-trimoxazole and azithromycin, monitoring adverse reactions and drug interaction in ART, patient training, follow-up after release from prison and finally, evaluation and monitoring of the HIV programs.

This was a quasi-experimental study that was conducted among 6,900 incarcerated individuals (median population during the intervention period) in the Great Tehran Prison that was selected as the intervention prison and 5,672 inmates (median population during the intervention period) in Alborz province prison as the control prison. Both prisons were chosen purposively. The intervention prison was a newly established prison without a previously structured health sector, especially for TB and HIV services. This enabled us to fully implement the intervention. Karaj prison was chosen as the control study prison due to its similar population size and the inmate's types of offenses. The investigators used all the questionnaires and forms related to the Iranian centers for disease control and prevention (CDC).

The study included three phases: pre-intervention, intervention and follow-up. The preintervention period lasted 22 months, from December 2011 to October 2013. The intervention period was from November 2013 to May 2014, followed by a one-month follow-up period during June 2014.

The intervention prison contains three separate units. First, all prisoners enter the "reception and identification unit (quarantine)" and then, according to their sentence status, are sent to the corresponding unit, which was nominated as number 1 and 2 . The control prison was Karaj prison, located in Alborz province. Similar to the intervention prison, inmates first enter the quarantine unit and are then sent to separate units based on their legal status. Both jails are male-only prisons. Inmates were incriminated for different crimes, but mostly robbery and drug trafficking.

TB active case finding (ACF) was implemented in the quarantine section, in both units 1 and 2. Three clinical staff members trained the prisoners about spotting the symptoms of TB infection in the units. In quarantine, all newly admitted prisoners were examined for the symptoms of TB. In unit 1 , healthcare staff continued the process of ACF, while in unit 2, the trained prisoners were assigned as healthcare lay providers to proceed with the process of $\mathrm{ACF}$. If any prisoner had TB symptoms, the process of diagnosis began and if TB was confirmed, care, treatment and follow-up procedures were initiated. Moreover, directly observed therapy (DOT) was simultaneously initiated for TB medication in prison. There was an active follow-up for released prisoners to link them to care and treatment services outside the prison in case of early release prior to the completion of TB treatment.

In both prisons, after inmate release or transfer, the follow-up was via a one-time phone call with outside healthcare providers to inform them about the continuation of inmate TB treatment. In the intervention prison, in addition to this routine follow-up, inmates were
Tuberculosis indicators in Iranian prisons 
JHR 34,6

actively observed and followed by a trained healthcare staff for continuity of services after being released or transferred. The released patients were followed up at least three times. Also, the telephone numbers and addresses of the patients were referred to the local health centers close to where they lived. We emphasized both the healthcare system and the patients adhering to the treatment.

The HIV case finding process continued in the prison with two different approaches; voluntary provider-initiated testing and voluntary client-initiated testing. Prisoners were asked to identify different risk factors of HIV infection and were given consultations. All people referred to the prison clinics were examined for the HIV infection if they had related risk factors. Once the result was negative, they were given the required information regarding prevention and risky behaviors. If, however, the test was positive, the process of care, treatment and follow-ups were initiated. If the test results were inconclusive, the prison inmates were requested to get retested after two months.

\section{Ethical considerations}

Oral and written informed consents were obtained from all the enrolled inmates in this study. Records were separately archived from the jail criminal record. The data was safely secured and kept confidential from the prisons' system and other inmates. Furthermore, the Institutional Review Board (IRB) from the Tehran University of Medical Sciences approved the project protocol (Ethics No. 9021598014).

\section{Statistical analysis}

The data were entered into and analyzed by STATA (version 14, College Station, TX, USA) using Pearson's Chi-squared, two-sample t-tests, paired t-test and the Kruskal-Wallis test to analyze the data and report the results.

\section{Results}

As presented in Table 1, the number of inmates with TB symptoms in the intervention prison who underwent a TB workup was four times more than that of the control prison (9.3 cases with TB symptoms assessed out of 1,000 inmates in the case prison vs 2.5 cases out of 1,000 inmates in the control prison each month). Such a difference was also significant in the intervention prison where the numbers of TB case finding were compared before and after the intervention period (9.3 cases with TB symptoms assessed out of 1,000 inmates in the intervention prison after intervention vs 2.0 cases out of 1,000 inmates before the intervention each month).

Due to an inadequate registration system, the healthcare workers in the control prison believed that $100 \%$ of possible TB cases had to undergo the sputum acid-fast bacillus (AFB) examination and all of them received the results of the AFB test. In the intervention prison, however, data were gathered by the newly developed registration system and $48 \%$ (215 out of 448 ) of possible infected inmates underwent an AFB examination. Of those 215 inmates, $92.6 \%$ (199 out of 215 inmates) had the results. Taking into account this fact, sputum exam uptake was still significantly higher in the intervention prison compared to the control prison (4.5 cases with AFB exams out of 1,000 inmates vs 2.5 in the control prison each month) and the intervention prison before the intervention (4.5 cases with AFB exams out of 1,000 inmates after intervention vs 2.0 before the intervention each month).

The patient findings significantly increased after intervention in the intervention prison (223.6 cases out of 100,000 inmates after vs 81.8 cases out of 100,000 inmates before intervention each year). However, the number of case findings was not significantly higher when compared with the control prison (223.6 cases out of 100,000 inmates in case prison vs 181.3 cases out of 100,000 inmates in the control prison each year, $p$-value $=0.69$ ). This was 
Table 1. Comparison of TB indicators in case and control prisons in different time periods 


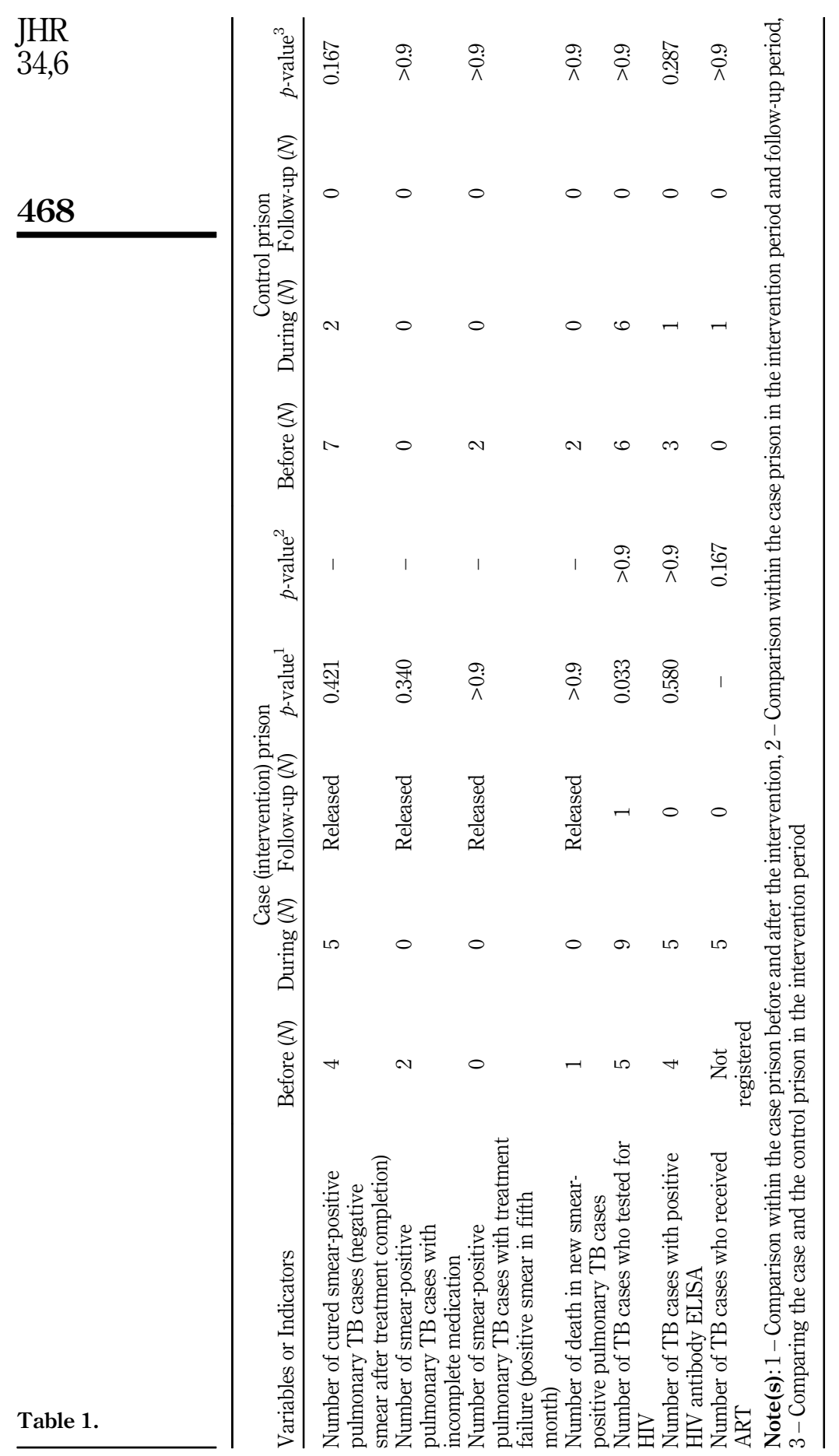


later attributed to the unfortunate lower quality of sputum specimens in the intervention prison, of which only $70 \%$ had the proper required quality. However, this data was not recorded in the control prison (Table 1).

During the one-month follow-up after the intervention, the evaluation rate of possible TB cases increased in the intervention prison. The total number of patients with available sputum AFB results did not significantly change; however, the total number of patientcollected sputum specimens detected cases of suspicious TB and the confirmed cases increased significantly in the intervention prison (Table 1).

After intervention implementation, all the sputum specimens in the intervention prison were microscopically tested as negative three times after the treatment and all the patients responded well to the treatment. However, before the intervention, the lack of a proper registration system in both prisons undermined any convincing results for treatment effectiveness as can be seen in Table 1 . Treatment interruption decreased from $29 \%$ to none in the intervention prison during the intervention. The number of cases of death among prisoners also decreased from $1(14.3 \%)$ to 0 . Although there was no incomplete medication in the control prison, the response to medication was not at an acceptable level (Table 1).

During the intervention period, only one out of $22(4.5 \%)$ inmates who claimed to have TB was confirmed as having a TB diagnosis. This data was not recorded in the intervention prison before the intervention and in the control prison (Table 1). In the intervention prison, the number of HIV positive-TB patients with a positive ELISA test increased from $5(50 \%)$ to $9(100 \%)$ patients $(p$-value $=0.033$ ). In the control prison, although all the TB cases were tested for HIV, the testing rate was significantly lower in possible TB cases compared to the intervention prison (Table 1).

\section{Discussion}

This study confirmed that the total number of people who were assessed for active TB significantly increased during the implementation of the guideline in the intervention prison compared to the control and intervention prison before the intervention. Due to the lack of a proper registration system, all numbers, including the number of individuals checked for sputum AFB, the number of individuals with available sputum AFB results and the total number of possible/suspicious TB cases with a confirming diagnosis, were similar to the numbers before intervention in both the intervention and control prisons. However, the number of suspected TB cases in the intervention prison was significantly more than the control prison before the implementation. In a study from one jail system in the United States, $79 \%$ of suspected TB patients had sputum samples collected, and smear results were missing for $13 \%$ and culture results were missing for $29 \%$ of patients with documented sputum collection [10]. Considering the duration of implementation (seven months) compared to the period before the implementation (22 months) and analyzing with time adjustment, the total number of suspicious/possible TB cases with a confirmed diagnosis was significantly higher after implementation in the intervention prison.

Although the number of screened TB suspects in the intervention prison was significantly higher than the control prison before implementation, the study showed no significant differences in the total number of diagnosed TB cases. This finding may suggest that our current screening tools are not sensitive enough and therefore, by using newer and better methods, this number might increase. Moreover, a study in sub-Saharan Africa showed that smear microscopy, chest radiography and clinical screening might be both slow and insensitive [4]. Also, another study in Brazil observed that two smears failed to detect TB in $74 \%$ of cases and they were positive through sputum culture. Among the smear-positive cases, only $75 \%$ were positive according to the first smear examination with the rest identified during the second smear evaluation. These findings suggest that one microscopy smear may be insufficient for active case detection. Additionally, because a quarter of the
Tuberculosis indicators in Iranian prisons 
JHR 34,6

cases were only diagnosed following a second culture evaluation, it would seem therefore that a collection of at least two specimens is recommended especially in performing mass screening in prisons [11]. This is similar to those results obtained from Colombian prisons [12]. A systematic review of screening practices suggested that using an application of chest $\mathrm{X}$-ray (CXRs) and symptom questionnaires, including the presence of a persistent cough, could increase the reliability of new TB case detection that could be detected by finding radiographic signs in unrecognized cases. In low- and middle-income countries, using CXRs, in addition to symptom questionnaires, will help with the increased sensitivity of screening algorithms [13]. However, 33\% of bacteriologically confirmed TB cases of prisoners in Zambia did not report any typical screening symptoms including cough, fever, weight loss or night sweats [14]. Further studies are needed to find more sensitive screening tools. Recently, the CDC recommended molecular tools to help diagnose TB cases [15].

With regard to medication follow-up that has a more precise registration, the effectiveness of the intervention was clearer. Due to the limited number of identified cases, statistical analysis was difficult. However, a smear exam was performed for all the smear-positive pulmonary TB patients in the intervention prison during treatment and the sputum tested negative for all the patients in the second month after treatment and all patients were cured in this prison. In the intervention prison, before implementation, these fractions were significantly lower. The rate of sputum smear conversion was significantly higher than in the control prison during implementation. These fractions were not acceptable in both intervention and control prisons before the intervention. In a larger sample size of patients, it is more likely that we would observe a significant statistical difference in the intervention prison. In our study, after implementing the guideline, there were no patients with incomplete medication, while about one-third of the patients did not complete their medications before the intervention. This finding emphasizes the importance of active service provision as suggested in our guidelines. On the other hand, the incidence of mortality in the intervention prison before the intervention was higher than in the control prison. This finding helps show the effectiveness of intervention to provide medication by AHSP.

Compared to the results of the study in Zambian prisons, which reported $91 \%$ of treatment initiation, $64 \%$ of treatment completion and $5 \%$ mortality and a study in Taiwan with $80.4 \%$ of treatment completion and $3.7 \%$ mortality and the study of the US jail systems with treatment completion of $17 \%$, AHSP seems to be more effective with $100 \%$ treatment initiation and completion and no mortality $[10,16,17]$. In the intervention prison, $4.5 \%$ of individuals claiming to have TB were confirmed as positive. Although this number was low, the importance of asking for TB histories cannot be ignored. However, this data was not available for both intervention and control prisons before the intervention, which was attributed to inadequate registration.

All TB patients of the intervention prison received the ELISA test for HIV in the intervention period while only half of the patients in this prison were tested before. All TB patients in the control prison were tested, although the number of suspected TB patients who were tested was significantly lower. In a study of US jail systems, HIV status was unknown or missing for $29 \%$ of inmates evaluated for TB and $52 \%$ of inmates with LTBI [10].

In general, improvement of prison conditions, nutrition, reduction in overcrowding and enhancing access to quality healthcare services can help control TB not only in prison but also in the general population. In addition, screening can play an important role in the early detection of TB patients, especially at the time of admission to prison, if enough resources are available $[18,19]$. Political and administrative commitment is required to ensure completion of treatment, which is important for a successful control program [18].

Different methods are applied to screen and manage TB in prisons across the globe. Educating both personnel and prisoners, training selected inmates (as peer educators), mass and community screening approaches, physical examinations, history taking, X-rays and 
two smear samples analyses are some of the recommended approaches in TB management [16]. The most common screening methods in European countries are the tuberculin skin test and a CXR following a positive tuberculin test upon entry and during imprisonment. TB patients were treated according to WHO recommendations in $90.9 \%$ of these countries, mostly free of charge and using DOT. Access to TB treatment after releasing inmates varies between European countries and ranged from 50 to $100 \%$ in countries with available data. Furthermore, $68.2 \%$ of these countries had guidelines for TB control and $59.1 \%$ of them provide education and TB awareness in prisons. The average cure rate was $32.9 \%$ [20]. In another approach in Taiwan, inmates with suspicious lesions on miniature films were notified to undergo follow-up CXR and sputum examination. But reliance on radiography versus sputum examination increased the risk of unreliable results [17]. Sputum smear microscopy specificity ranges from 93 to $100 \%$. CXR is more accurate than symptom screening [5].

Various methods have been used in Iran. In a study in the Mazandaran province prisons, a symptom-based questionnaire was filled out for prisoners at the first step, then prisoners with chronic cough, past medical history or family history of TB and HIV-positive prisoners underwent a sputum smear analysis [21]. In another study on Iranian prisoners with a history of injection drug use, a purified protein derivative (PPD) skin test was performed and individuals with suggestive symptoms of TB were examined through sputum examination and, if necessary, X-ray [22].

Overall, worldwide, according to a systematic review, the most common screening tool was symptom questionnaires $(63.5 \%)$, mostly reporting the presence of a persistent cough. Microscopic analyses of sputum with Ziehl-Neelsen staining and solid culture were the most frequently combined diagnostic methods $(21.2 \%)$. CXR and tuberculin skin tests were used by 73.1 and $50 \%$, respectively, as either a screening and/or diagnostic tool. Inaccurate diagnostic algorithms and inadequate laboratory facilities were the major limitations of a proper TB control program in prisons. The most common recommendation for increasing success in TB control programs is to increase screening frequency [13].

\section{Conclusions}

This clinical guideline has significantly improved the defined indicators in the intervention prison in comparison with the control prison. Therefore, we recommend future multicenter studies with the implementation of a guideline based on AHSP in all prisons of Iran, alongside establishing a reliable national registration system that can enable researchers to perform further nation-wide studies with longer follow-up periods in both male and female prisons and more accurately address possible confounders of the results. The method used in this study provides significant opportunities to detect and follow up on the treatment of HIVpositive and TB-positive inmates. In addition, the development and implementation of similar guidelines for other diseases with a high burden among prisoners are recommended and can lead to significant enhancement in the quality of care.

\footnotetext{
Limitation

A major limitation was the lack of female prisoners in our samples since female sampling is very difficult in the Middle East region due to cultural obstacles and also because the majority of Iranian inmates are males. Another limitation was an inadequate registry system in the control prison, resulting in lower or less reliable numbers in a few variables, which might have led to a level of uncertainty in some of the judgments made. However, this limitation was inevitable in the current study and this collateral finding signifies the importance of highly validated protocols with proper registration of results in prison systems. Further multicenter studies in other Iranian prisons can demonstrate the effects of the implemented guideline more accurately.
} 
JHR 34,6

Furthermore, rapidly transferring patients between the different units caused difficulties in screening, diagnosis, testing, treatment and follow-ups. In addition, some prisoners with highrisk behaviors related to HIV and positive rapid diagnostic tests were released from prison before the confirmatory tests (ELISA, Western blot) were completed. As the main index for being HIV-positive approved by the Ministry of Health was the Western blot test, we faced serious hardship in following up those patients outside the prison. Although recently, the Western blot has been removed from the algorithm of HIV diagnosis confirmation.

\section{References}

1. Golrokhi R, Farhoudi B, Taj L, Pahlaviani FG, Mazaheri-Tehrani E, Cossarizza A, SeyedAlinaghi $\mathrm{S}$, Mohraz M, Voltarelli FA. HIV prevalence and correlations in prisons in different regions of the World: a review article. Open AIDS J. 2018; 12: 81-92.

2. Biadglegne F, Rodloff AC, Sack U. Review of the prevalence and drug resistance of tuberculosis in prisons: a hidden epidemic. Epidemiol Infect. 2015 Apr; 143(5): 887-900.

3. Baussano I, Williams BG, Nunn P, Beggiato M, Fedeli U, Scano F. Tuberculosis incidence in prisons: a systematic review. PLoS Med. 2010 Dec 21; 7(12): e1000381.

4. Reid SE, Topp SM, Turnbull ER, Hatwiinda S, Harris JB, Maggard KR, Roberts ST, Krüüner A, Morse JC, Kapata N, Chisela C, Henostroza G. Tuberculosis and HIV control in sub-Saharan African prisons: 'thinking outside the prison cell'. J Infect Dis. 2012 May 15; 205(Suppl 2): S265-73.

5. Kranzer K, Afnan-Holmes H, Tomlin K, Golub JE, Shapiro AE, Schaap A, Corbett EL, Lönnroth K, Glynn JR. The benefits to communities and individuals of screening for active tuberculosis disease: a systematic review. Int J Tuberc Lung Dis. 2013 Apr; 17(4): 432-46.

6. Dara M, Acosta CD, Melchers NV, Al-Darraji HA, Chorgoliani D, Reyes H, Centis R, Sotgiu G, D’Ambrosio L, Chadha SS, Migliori GB. Tuberculosis control in prisons: current situation and research gaps. Int J Infect Dis. 2015 Mar; 32: 111-7.

7. Bjørngaard JH, Rustad $\AA \mathrm{B}$, Kjelsberg E. The prisoner as patient-a health services satisfaction survey. BMC Health Serv Res. 2009 Sep 28; 9: 176.

8. Farhoudi B, SeyedAlinaghi S, Hosseini M, Pahlaviani FG, Firouzeh MM, Shahbazi M, Mohraz M. Prevalence of tuberculosis in a prison in tehran by active case finding. Infect Disord Drug Targets. 2019; 19(2): 167-170.

9. Farhoudi B, SeyedAlinaghi S, Mohraz M, Golrokhy R, Farnia M, Shahbazi M, Alasvand R, Ebrahimi B, Esfehani J, Tashakoriyan M. Revision and implementation of 'clinical guideline for tuberculosis and HIV in prisons', Great Tehran prison, Iran. Infect Disord Drug Targets. 2018; 18(1): 72-80.

10. Reichard AA, Lobato MN, Roberts CA, Bazerman LB, Hammett TM. Assessment of tuberculosis screening and management practices of large jail systems. Public Health Rep. 2003 Nov-Dec; 118(6): 500-7.

11. Carbone A, Paião D, Sgarbi R, Lemos E, Cazanti R, Ota M, Junior AL, Bampi JV, Elias VP, Simionatto S, Motta-Castro AR, Pompílio MA, de Oliveira SM, Ko AI, Andrews JR, Croda J. Active and latent tuberculosis in Brazilian correctional facilities: a cross-sectional study. BMC Infect Dis. 2015 Jan 22; 15: 24.

12. Rueda ZV, Lopez L, Velez LA, Marin D, Giraldo MR, Pulido H, Orozco LC, Montes F, Arbeláez MP. High incidence of tuberculosis, low sensitivity of current diagnostic scheme and prolonged culture positivity in four Colombian prisons. A cohort study. PLoS One. 2013 Nov 21; 8(11): e80592.

13. Vinkeles Melchers NV, van Elsland SL, Lange JM, Borgdorff MW, van den Hombergh J. State of affairs of tuberculosis in prison facilities: a systematic review of screening practices and recommendations for best TB control. PLoS One. 2013; 8(1): e53644.

14. Henostroza G, Topp SM, Hatwiinda S, Maggard KR, Phiri W, Harris JB, Krüüner A, Kapata N, Ayles H, Chileshe C, Reid SE The high burden of tuberculosis (TB) and human immunodeficiency virus (HIV) in a large Zambian prison: a public health alert. PLoS One. 2013; 8(8): e67338. 
15. Lewinsohn DM, Leonard MK, LoBue PA, Cohn DL, Daley CL, Desmond E, Keane J, Lewinsohn DA, Loeffler AM, Mazurek GH, O’Brien RJ, Pai M, Richeldi L, Salfinger TM, Sterling TR, Warshauer DM, Woods GL. Official American thoracic society/infectious diseases society of America/centers for disease control and prevention clinical practice guidelines: diagnosis of tuberculosis in adults and children. Clin Infect Dis. 2017 Jan 15; 64(2): e1-e33.

16. Maggard KR, Hatwiinda S, Harris JB, Phiri W, Kruuner A, Kaunda K, Topp SM, Kapata N, Ayles H, Chileshe C, Henostroza G, Reid SE. Screening for tuberculosis and testing for human immunodeficiency virus in Zambian prisons. Bull World Health Organ. 2015 Feb 1; 93(2): 93-101.

17. Chen-Yuan Chiang CJH, Hsu PK, Suo J, Lin TP. Pulmonary tuberculosis in the Taiwanese prison population. J Formos Med Assoc. 2002 Aug; 101(8): 537-41.

18. Rudi Coninx DM, Reyes H, Grzemska M. Tuberculosis in prisons in countries with high prevalence. BMJ. 2010; 320: 440-2.

19. Banu S, Hossain A, Uddin MK, Uddin MR, Ahmed T, Khatun R, Mahmud AM, Hyder KA, Lutfor AB, Karim MS, Zaman K, Khan MA, Barua PC, Luby SP. Pulmonary tuberculosis and drug resistance in dhaka central jail, the largest prison in Bangladesh. PLoS One. 2010 May 21; 5(5): e10759.

20. Aerts A, Hauer B, Wanlin M, Veen J. Tuberculosis and tuberculosis control in European prisons. Int J Tuberc Lung Dis. 2006 Nov; 10(11): 1215-23.

21. Moosazadeh M, Parsaei M, Ahmadi M, Jalahi H. TB prevalence in prisons of Mazandaran province in 2008:breif report. Rafsanjan University of Medical Sciences J. 2008; 10(4): 309-16.

22. Ahmad Seyed Alinaghi S, Farhoudi B, Hosseini M, Mohammadi Firouzeh M, Mohraz M, Tashakoriyan M. Quickness of HIV and tuberculosis diagnostic procedures in prison of tehran, Iran. Infect Disord Drug Targets. 2016; 16(2): 109-12.

\section{Corresponding author}

Behnam Farhoudi can be contacted at: b_farhoudi@yahoo.com
Tuberculosis indicators in Iranian prisons 